\title{
Inflation nach Corona: Sind die Sorgen berechtigt?
}

Im August 2021 wird die deutsche Inflationsrate voraussichtlich auf 3,9\% steigen. Ein Teil davon dürfte einmaligen oder vorübergehenden Effekten geschuldet sein. Hierzu zählen in Deutschland vor allem die Rücknahme der Mehrwertsteuersenkung, die Ausweitung der $\mathrm{CO}_{2}$-Bepreisung sowie Preisanstiege durch die Knappheit von Rohstoffen und Vorprodukten. Dennoch mag es strukturelle Gründe für eine steigende Inflation geben. So werden zum Teil die lockere Geldpolitik und das neue Inflationsziel der EZB für höhere Inflationsrisiken verantwortlich gemacht. Außerdem führt die expansive Fiskalpolitik der neuen US-Administration zu höheren Preissteigerungsraten in den USA. Ob die Möglichkeit einer Lohn-Preis-Spirale besteht und ob die Inflationsmessung verbesserungsfähig ist, wird ebenfalls im Rahmen dieses Zeitgesprächs diskutiert.

Inflationsangst in Deutschland - Vorsicht vor zu engem Blick auf Konjunktur und Geldmenge Johannes Mayr, Eyb \& Wallwitz Vermögensmanagement GmbH, München.

Zielkonforme Preisentwicklung im Euroraum: Implikationen für die deutsche Wirtschaftspolitik

Peter Bofinger, Universität Würzburg.

Inflation 2021: kein geldpolitischer Handlungsbedarf

Silke Tober, Institut für Makroökonomie und Konjunkturforschung (IMK), Düsseldorf.

Auch die Konsumentenpreisinflation dürfte auf längere Zeit steigen

Gunther Schnabl, Universität Leipzig.

Tim Florian Sepp, Universität Leipzig.

Ist das neue Inflationsziel der EZB noch zeitgemäß?

Friedrich L. Sell, Universität der Bundeswehr München, Neubiberg. 